\title{
A Rapid Assay to Assess Nitrification Inhibition Using a Panel of Bacterial Strains and Partial Least Squares Modeling
}

Patrick Morkus, Mehdi Zolfaghari, Salman Alizadeh Kordkandi, Jake Nease, Carlos D. M. Filipe, David R. Latulippe*

Department of Chemical Engineering, McMaster University, 1280 Main Street West, Hamilton, Ontario, L8S 4L7, Canada

* Corresponding author; phone number: 1-905-525-9140 extension 24011; e-mail address: latulippe@mcmaster.ca

Supporting Information: 13 pages, 6 figures, 4 tables 


\begin{tabular}{|c|c|c|c|c|c|c|c|c|}
\hline \multirow{2}{*}{$\begin{array}{c}\text { WW } \\
\text { Sample }\end{array}$} & \multirow{2}{*}{$\begin{array}{c}\text { SNR Outcome } \\
\% \text { SNR difference }\end{array}$} & \multicolumn{7}{|c|}{ Bacterial Strain Fluorescence Signal } \\
\hline & & Nitrospira & N. europaea & E. coli & B. subtilis & B. cereus & S. saprophyticus & S. laurentii \\
\hline 1 & $0.58($ Fail $)$ & 3072 & 4279 & 5984 & 18258 & 9682 & 4956 & 22007 \\
\hline 2 & 3.93 (Fail) & 3194 & 4719 & 8063 & 17817 & 10221 & 4618 & 22183 \\
\hline 3 & 5.65 (Fail) & 3137 & 5412 & 5244 & 19517 & 10289 & 4592 & 21800 \\
\hline 4 & 6.35 (Fail) & 3082 & 4278 & 7717 & 16312 & 9508 & 3997 & 19418 \\
\hline 5 & 15.45 (Fail) & 3406 & 4867 & 5774 & 19640 & 12206 & 5580 & 22162 \\
\hline 6 & -12.58 (Pass) & 2756 & 4350 & 6198 & 18142 & 8855 & 3976 & 21993 \\
\hline 7 & 1.51 (Fail) & 3411 & 4897 & 7727 & 19716 & 10913 & 4237 & 21643 \\
\hline 8 & -13.53 (Pass) & 2561 & 4425 & 6694 & 18781 & 8964 & 4725 & 21569 \\
\hline 9 & 5.45 (Fail) & 3380 & 5358 & 6745 & 19027 & 10007 & 5551 & 23272 \\
\hline 10 & -1.43 (Pass) & 2882 & 4414 & 6116 & 17844 & 8724 & 4906 & 21163 \\
\hline 11 & -9.66 (Pass) & 2731 & 4419 & 5571 & 19038 & 9453 & 4855 & 22923 \\
\hline 12 & -3.89 (Pass) & 2869 & 4362 & 6013 & 19312 & 9250 & 5041 & 22038 \\
\hline 13 & 13.64 (Fail) & 3100 & 5048 & 7010 & 17058 & 9195 & 4851 & 23851 \\
\hline 14 & -7.60 (Pass) & 2747 & 4383 & 5902 & 18322 & 8669 & 4840 & 24482 \\
\hline 15 & -1.17 (Pass) & 3220 & 4876 & 5665 & 19849 & 9936 & 5303 & 23295 \\
\hline 16 & -6.84 (Pass) & 2734 & 4517 & 6011 & 19352 & 9103 & 4868 & 21432 \\
\hline 17 & 29.66 (Fail) & 2937 & 3986 & 7044 & 16750 & 8003 & 4294 & 22108 \\
\hline 18 & 26.01 (Fail) & 3029 & 4035 & 9438 & 13754 & 7863 & 4064 & 21836 \\
\hline 19 & 0.23 (Fail) & 3349 & 4531 & 6034 & 18156 & 10027 & 5118 & 22834 \\
\hline 20 & 0.90 (Fail) & 2880 & 4134 & 8630 & 20688 & 11062 & 4475 & 23363 \\
\hline 21 & 5.66 (Fail) & 3471 & 3955 & 6802 & 18051 & 8832 & 5102 & 23121 \\
\hline 22 & 2.00 (Fail) & 2938 & 3217 & 8747 & 19146 & 9729 & 4538 & 22602 \\
\hline 23 & -7.48 (Pass) & 2611 & 4079 & 5192 & 17903 & 9216 & 4572 & 22942 \\
\hline 24 & 1.24 (Fail) & 2497 & 3676 & 6709 & 18076 & 9910 & 4532 & 22226 \\
\hline 25 & 3.38 (Fail) & 2972 & 3815 & 6018 & 19141 & 10004 & 4798 & 22130 \\
\hline 26 & -15.54 (Pass) & 3024 & 3713 & 6653 & 20500 & 8959 & 5002 & 22556 \\
\hline 27 & -6.04 (Pass) & 2619 & 2492 & 6203 & 19902 & 8856 & 4798 & 24028 \\
\hline 28 & 13.95 (Fail) & 2778 & 2583 & 6942 & 19085 & 10687 & 5834 & 26079 \\
\hline
\end{tabular}

Table S1. Comparison of results for 28 industrial WW samples for the SNR test (highlighted in gray) and the CBB panel fluorescence data (highlighted in orange). The error (standard deviation) associated with the fluorescence signals was between 1-5\% based on duplicate testing. Duplicate analysis of WW sample 2 and 18 showed that the $\%$ SNR Difference values and their errors were approximately $4 \pm 2$ and $26 \pm 5$, respectively. 


\begin{tabular}{|c|c|c|c|c|c|c|c|c|c|}
\hline \multirow[b]{2}{*}{$\begin{array}{c}\text { WW } \\
\text { Sample }\end{array}$} & \multirow{2}{*}{$\begin{array}{c}\text { SNR Outcome } \\
\% \text { SNR } \\
\text { difference }\end{array}$} & \multicolumn{8}{|c|}{ Gas Chromatography-Mass Spectrometry (normalized integral peak area) } \\
\hline & & $\begin{array}{l}\text { Butanoic } \\
\text { acid }\end{array}$ & $\begin{array}{l}\text { Ethylene } \\
\text { glycol }\end{array}$ & $\begin{array}{c}\text { Propylene } \\
\text { glycol }\end{array}$ & $\beta$-Eudesmol & $\begin{array}{l}\text { Heptanoic } \\
\text { acid }\end{array}$ & p-Anisic acid & $\begin{array}{l}\text { Triethylene } \\
\text { glycol butyl } \\
\text { ether }\end{array}$ & 1-Octanol \\
\hline 1 & 0.58 & 1.154 & 0 & 0 & 0 & 2.063 & 0 & 0 & 0 \\
\hline 2 & 3.93 & 24.463 & 0 & 0 & 0 & 6.908 & 0 & 9.981 & 0 \\
\hline 3 & 5.65 & 0 & 0 & 0 & 0 & 0 & 0 & 0.984 & 0 \\
\hline 4 & 6.35 & 0 & 0 & 0 & 0 & 0 & 0 & 0 & 0 \\
\hline 6 & -12.58 & 0 & 0.195 & 0 & 0 & 0 & 0 & 0.227 & 0.296 \\
\hline 7 & 1.51 & 0 & 0 & 0.540 & 1.409 & 0 & 0 & 0.514 & 0.371 \\
\hline 8 & -13.53 & 0 & 0 & 0 & 0 & 0 & 0 & 0.608 & 0.362 \\
\hline 9 & 5.45 & 0 & 0.610 & 0.505 & 0.758 & 0 & 0 & 0.286 & 0.389 \\
\hline 10 & -1.43 & 0 & 0 & 0 & 0 & 0 & 0 & 1.512 & 0 \\
\hline 11 & -9.66 & 0 & 0 & 0 & 0 & 0 & 0 & 0 & 0 \\
\hline 12 & -3.89 & 0 & 0 & 0 & 0 & 10.989 & 0 & 0 & 0 \\
\hline 13 & 13.64 & 0 & 0 & 0 & 0 & 5.092 & 0 & 5.855 & 0 \\
\hline 14 & -7.60 & 0 & 0 & 0 & 0 & 0 & 0 & 0 & 0 \\
\hline 16 & -6.84 & 0 & 0 & 1.445 & 1.793 & 0 & 0 & 0 & 0 \\
\hline 20 & 0.90 & 0 & 0 & 0 & 0 & 1.409 & 0 & 0 & 0 \\
\hline 22 & 2.00 & 0.494 & 0 & 0 & 0 & 0 & 0 & 0 & 0 \\
\hline 24 & 1.24 & 0 & 0 & 1.567 & 0 & 0 & 0.250 & 0 & 0 \\
\hline 27 & -6.04 & 0 & 0 & 0 & 0 & 0 & 0 & 0 & 0 \\
\hline 28 & 13.95 & 0 & 0 & 0 & 0 & 0 & 0 & 0 & 0 \\
\hline
\end{tabular}

Table S2. Gas Chromatography-Mass Spectrometry (GC-MS) and \% SNR difference. The GC-MS values (highlighted in green) were measured using the procedure in section 2.4. Each value corresponds to the integral peak area normalized by integral peak area of the internal standard; zero values are used to indicate that the corresponding chemical compound was not detected. The \% SNR difference values (highlighted in gray) were measured using the procedure in section 2.3. Table continued on next page. 


\begin{tabular}{|c|c|c|c|c|c|c|c|c|c|c|c|}
\hline \multirow{2}{*}{$\begin{array}{c}\text { WW } \\
\text { Sample }\end{array}$} & \multicolumn{10}{|c|}{ Gas Chromatography-Mass Spectrometry (normalized integral peak area) } & \multirow[b]{2}{*}{$\begin{array}{c}\text { Decanoi } \\
\text { c acid }\end{array}$} \\
\hline & $\begin{array}{l}\text { Ethylhexanoi } \\
\text { c acid }\end{array}$ & $\begin{array}{c}2- \\
\text { Phenylethano } \\
1\end{array}$ & $\begin{array}{l}\text { Nonanoi } \\
\text { c acid }\end{array}$ & $\begin{array}{c}\text { Isoeugeno } \\
1\end{array}$ & Phenol & $\begin{array}{c}\text { (E)- } \\
\text { Cinnami } \\
\text { c acid }\end{array}$ & $\begin{array}{l}\text { Pentanoi } \\
\text { c acid }\end{array}$ & $\begin{array}{c}2- \\
\text { Butoxyethano } \\
1\end{array}$ & $\begin{array}{l}\text { Hexanoi } \\
\text { c acid }\end{array}$ & $\begin{array}{l}\text { Benzyl } \\
\text { alcohol }\end{array}$ & \\
\hline 1 & 1.632 & 0.418 & 0 & 0 & 0.274 & 0 & 0.593 & 8.277 & 0.460 & 0.663 & 0 \\
\hline 2 & 5.882 & 0 & 2.739 & 0 & 3.868 & 0 & 0 & 14.682 & 3.335 & 3.397 & 0 \\
\hline 3 & 5.055 & 0 & 0 & 0 & 0 & 0 & 0 & 5.077 & 0 & 1.191 & 0 \\
\hline 4 & 50.659 & 0 & 0 & 0 & 0 & 0 & 0 & 16.241 & 0 & 12.898 & 6.463 \\
\hline 6 & 0 & 0 & 0 & 0 & 0 & 0 & 0 & 0 & 0 & 0 & 0 \\
\hline 7 & 0 & 0 & 0 & 0.849 & 0 & 0 & 0.258 & 6.570 & 0 & 5.410 & 0 \\
\hline 8 & 0 & 0 & 0 & 0 & 0.255 & 0 & 0.332 & 2.310 & 0 & 0.231 & 0 \\
\hline 9 & 0 & 0.388 & 0 & 0.761 & 0 & 0 & 0.370 & 0 & 0 & 1.732 & 0 \\
\hline 10 & 0 & 0 & 0 & 0 & 0 & 0 & 0 & 7.001 & 0 & 2.010 & 0 \\
\hline 11 & 0 & 0 & 0 & 0 & 0 & 0 & 0 & 3.090 & 0 & 0 & 0 \\
\hline 12 & 19.567 & 0 & 4.345 & 0 & 0 & 0 & 0 & 28.942 & 0 & 0 & 0 \\
\hline 13 & 0 & 0 & 2.058 & 0 & 0 & 0 & 0 & 0 & 0 & 4.856 & 0 \\
\hline 14 & 0 & 0 & 0 & 0 & 1.271 & 0 & 0 & 4.556 & 0 & 0 & 0 \\
\hline 16 & 0 & 0 & 0 & 0 & 0 & 0 & 0 & 5.686 & 0 & 4.419 & 0 \\
\hline 20 & 1.532 & 0 & 0.566 & 0 & 1.494 & 0 & 0 & 0 & 0 & 1.223 & 0 \\
\hline 22 & 1.779 & 0 & 0.304 & 0 & 0.324 & 0 & 0 & 2.550 & 0.489 & 1.251 & 0 \\
\hline 24 & 2.287 & 0 & 0 & 0 & 0 & 0.216 & 0 & 2.043 & 0.673 & 1.619 & 0 \\
\hline 27 & 0 & 0 & 0 & 0 & 0.646 & 0 & 0 & 4.442 & 0 & 1.462 & 0 \\
\hline 28 & 32.435 & 0 & 12.503 & 0 & 0 & 0 & 0 & 60.597 & 0 & 15.977 & 0 \\
\hline
\end{tabular}

...Table S2. Continued on next page. 


\begin{tabular}{|c|c|c|c|c|c|c|c|c|c|c|c|}
\hline \multirow[b]{2}{*}{$\begin{array}{c}\text { WW } \\
\text { Sample }\end{array}$} & \multicolumn{11}{|c|}{ Gas Chromatography-Mass Spectrometry (normalized integral peak area) } \\
\hline & $\begin{array}{l}\text { Octanoic } \\
\text { acid }\end{array}$ & $\begin{array}{l}\text { Benzoic } \\
\text { acid }\end{array}$ & $\begin{array}{c}\text { Diethylene } \\
\text { glycol } \\
\text { butyl ether }\end{array}$ & $\begin{array}{l}\text { Phenoxy } \\
\text { ethanol }\end{array}$ & $\begin{array}{l}\text { Cyclohex } \\
\text { anamine }\end{array}$ & $\begin{array}{l}\text { Sebacic } \\
\text { acid }\end{array}$ & $\begin{array}{l}\text { Undecane } \\
\text { dioic acid }\end{array}$ & $\begin{array}{l}\text { Dodecane } \\
\text { dioic acid }\end{array}$ & 1-Hexanol & $\begin{array}{c}1- \\
\text { Phenyletha } \\
\text { nol }\end{array}$ & $\begin{array}{l}\text { Ricinoleic } \\
\text { acid }\end{array}$ \\
\hline 1 & 2.755 & 0.711 & 4.619 & 0 & 0 & 1.095 & 3.347 & 2.535 & 0 & 0 & 0 \\
\hline 2 & 13.015 & 6.125 & 16.021 & 0 & 0 & 5.553 & 14.980 & 12.162 & 0 & 0 & 0 \\
\hline 3 & 5.322 & 1.995 & 3.210 & 0 & 0 & 2.873 & 1.424 & 1.250 & 0 & 0 & 0 \\
\hline 4 & 9.829 & 5.941 & 13.728 & 0 & 0 & 4.351 & 6.532 & 5.270 & 0 & 0 & 0 \\
\hline 6 & 0 & 0 & 0.400 & 0 & 0 & 0 & 0 & 0 & 0 & 0 & 0 \\
\hline 7 & 0 & 0 & 3.233 & 0 & 0 & 0 & 0 & 0 & 0 & 3.017 & 0 \\
\hline 8 & 0 & 0 & 3.302 & 0 & 0 & 0 & 0 & 0 & 0 & 0 & 0 \\
\hline 9 & 0 & 0 & 2.043 & 0.855 & 0.360 & 0 & 0 & 0 & 0 & 0.302 & 0 \\
\hline 10 & 0 & 0 & 1.808 & 0 & 0 & 0 & 0 & 0 & 0.627 & 1.440 & 0 \\
\hline 11 & 0 & 0 & 1.007 & 0 & 0 & 0 & 0 & 0 & 0 & 0 & 0 \\
\hline 12 & 31.668 & 5.025 & 18.775 & 0 & 0 & 13.513 & 32.021 & 35.599 & 0 & 0 & 0 \\
\hline 13 & 8.967 & 4.682 & 10.111 & 0 & 0 & 3.037 & 8.292 & 6.947 & 0 & 0 & 0 \\
\hline 14 & 0 & 0 & 2.090 & 0 & 0 & 0 & 0 & 0 & 0 & 0 & 0 \\
\hline 16 & 0 & 0 & 2.395 & 2.470 & 0 & 0 & 0 & 0 & 0 & 0.921 & 0 \\
\hline 20 & 2.421 & 0.697 & 0.809 & 0 & 0 & 0.387 & 2.009 & 1.056 & 0 & 0 & 0 \\
\hline 22 & 2.616 & 0.876 & 0.997 & 0 & 0.379 & 0.613 & 1.290 & 1.207 & 0.574 & 0 & 0.563 \\
\hline 24 & 2.336 & 3.027 & 0.870 & 0 & 0 & 0.455 & 4.164 & 2.067 & 0 & 0 & 0 \\
\hline 27 & 0 & 0 & 1.279 & 0 & 0 & 0 & 0 & 0 & 0 & 0.632 & 0 \\
\hline 28 & 72.978 & 16.239 & 32.955 & 0 & 0 & 43.884 & 56.705 & 48.564 & 0 & 0 & 0 \\
\hline
\end{tabular}

...Table S2. Continued on next page. 


\begin{tabular}{|c|c|c|c|c|c|c|c|c|c|c|c|}
\hline \multirow[b]{2}{*}{$\begin{array}{c}\text { WW } \\
\text { Sample }\end{array}$} & \multicolumn{11}{|c|}{ Gas Chromatography-Mass Spectrometry (normalized integral peak area) } \\
\hline & Linalool & $\begin{array}{c}\text { L- } \alpha- \\
\text { Terpineol }\end{array}$ & $\begin{array}{l}\text { Dicyclohe } \\
\text { xylamine }\end{array}$ & $\begin{array}{l}\text { Palmitic } \\
\text { Acid }\end{array}$ & $\begin{array}{l}\text { Carbodi } \\
\text { imide }\end{array}$ & $\begin{array}{l}\text { Dibutyl } \\
\text { phthalate }\end{array}$ & $\begin{array}{c}3,6,9,12,15- \\
\text { Pentaoxahex } \\
\text { adecan-1-ol }\end{array}$ & Cresol & $\begin{array}{c}2- \\
\text { Butoxyethyl }\end{array}$ & $\begin{array}{l}\text { Cinnamyl } \\
\text { Alcohol }\end{array}$ & $\begin{array}{c}\text { 3- } \\
\text { Hydroxyiso } \\
\text { valeric acid }\end{array}$ \\
\hline 1 & 0 & 0 & 0.899 & 0 & 0 & 0 & 0 & 0 & 0 & 0 & 0 \\
\hline 2 & 0 & 0 & 3.752 & 0 & 0 & 0 & 0 & 0 & 0 & 0 & 0 \\
\hline 3 & 0 & 0 & 0 & 0 & 0 & 0 & 0 & 0 & 0 & 0 & 0 \\
\hline 4 & 0 & 0 & 0 & 0 & 0 & 0 & 0 & 0 & 0 & 0 & 0 \\
\hline 6 & 0 & 0 & 0 & 0 & 0.181 & 0.146 & 0 & 0 & 0 & 0 & 0 \\
\hline 7 & 0.680 & 1.263 & 0.687 & 0 & 0.171 & 0 & 0 & 0 & 0 & 0.306 & 0 \\
\hline 8 & 0 & 0 & 1.284 & 0 & 0.182 & 0 & 0.255 & 0 & 0 & 0 & 0 \\
\hline 9 & 0.645 & 0.958 & 1.691 & 0.114 & 0 & 0 & 0 & 0 & 5.425 & 0.212 & 0 \\
\hline 10 & 1.652 & 0 & 0 & 0 & 0 & 0 & 0 & 0 & 0 & 0 & 0 \\
\hline 11 & 0 & 0 & 0 & 0 & 0 & 0 & 0 & 0.248 & 0 & 0 & 0 \\
\hline 12 & 0 & 0 & 8.415 & 0 & 0 & 0 & 0 & 0 & 0 & 0 & 0 \\
\hline 13 & 0 & 0 & 2.220 & 0 & 0 & 0 & 0 & 0 & 0 & 0 & 0 \\
\hline 14 & 0 & 0 & 0 & 0 & 0 & 0 & 0 & 4.496 & 0 & 0 & 0 \\
\hline 16 & 2.346 & 0 & 2.480 & 0 & 0 & 0 & 0 & 0 & 0 & 0 & 7.129 \\
\hline 20 & 0 & 0 & 0.387 & 0 & 0 & 0 & 0 & 0 & 3.800 & 0 & 0 \\
\hline 22 & 0 & 0 & 0 & 0 & 0 & 0 & 0 & 0 & 0 & 0 & 0 \\
\hline 24 & 0 & 0 & 0 & 0 & 0 & 0 & 0 & 0 & 0 & 0 & 0 \\
\hline 27 & 0 & 0 & 0 & 0 & 0 & 0 & 0 & 2.002 & 0 & 0 & 0 \\
\hline 28 & 0 & 0 & 0 & 0 & 0 & 0 & 0 & 0 & 0 & 0 & 0 \\
\hline
\end{tabular}

...Table S2. Continued on next page. 


\begin{tabular}{|c|c|c|c|c|c|c|c|c|c|c|}
\hline \multirow[b]{2}{*}{$\begin{array}{c}\text { WW } \\
\text { Sample }\end{array}$} & \multicolumn{10}{|c|}{ Gas Chromatography-Mass Spectrometry (normalized integral peak area) } \\
\hline & Carbitol & $\begin{array}{c}2,3- \\
\text { Butanediol }\end{array}$ & $\begin{array}{c}2- \\
\text { Ethylhexan } \\
\text { ol }\end{array}$ & $\begin{array}{c}\text { 5- } \\
\text { Phenylnonane }\end{array}$ & $\begin{array}{c}2,6- \\
\text { Dimethyl } \\
\text { phenol }\end{array}$ & $\begin{array}{c}\text { Cyclohexan } \\
\text { ol }\end{array}$ & 2-Butanol & $\begin{array}{c}\text { Trifluoroac } \\
\text { etamide }\end{array}$ & $\begin{array}{l}\text { Dehydroabie } \\
\text { tic acid }\end{array}$ & $\begin{array}{c}\mathrm{p}- \\
\text { Chlorocresol }\end{array}$ \\
\hline 1 & 0 & 0 & 0 & 0 & 0 & 0 & 0 & 0 & 0.758 & 0 \\
\hline 2 & 0 & 0 & 0 & 0 & 0 & 0 & 0 & 0 & 0 & 2.345 \\
\hline 3 & 0 & 0 & 0 & 0 & 0 & 0 & 0 & 14.769 & 0 & 0 \\
\hline 4 & 0 & 0 & 0 & 0 & 0 & 0 & 0 & 0 & 0 & 0 \\
\hline 6 & 0 & 0 & 0 & 0 & 0 & 0 & 0 & 0 & 0 & 0 \\
\hline 7 & 0.311 & 0.945 & 0 & 0 & 0 & 0 & 0 & 0 & 0 & 0 \\
\hline 8 & 0 & 0 & 0 & 0 & 0 & 0 & 0 & 0 & 0 & 0 \\
\hline 9 & 0 & 0 & 0 & 0 & 0 & 0 & 0 & 0 & 0 & 0 \\
\hline 10 & 0 & 1.285 & 0 & 0 & 0 & 0.555 & 1.796 & 0 & 0 & 0 \\
\hline 11 & 0 & 0 & 0.672 & 0.304 & 0 & 0 & 0 & 0 & 0 & 0 \\
\hline 12 & 0 & 0 & 0.185 & 0 & 0 & 0 & 0 & 0 & 0 & 0 \\
\hline 13 & 0 & 0 & 0 & 0 & 0 & 0 & 0 & 0 & 0 & 1.555 \\
\hline 14 & 0 & 0 & 0 & 0 & 1.846 & 0 & 0 & 0 & 0 & 0 \\
\hline 16 & 0 & 0 & 0 & 0 & 0 & 0 & 0 & 0 & 0 & 0 \\
\hline 20 & 1.081 & 0 & 0 & 0 & 0 & 0 & 0 & 0 & 0 & 0 \\
\hline 22 & 1.347 & 0 & 0 & 0 & 0 & 0 & 0 & 0 & 0 & 0 \\
\hline 24 & 1.188 & 0 & 0 & 0 & 0 & 0 & 0 & 0 & 0 & 0 \\
\hline 27 & 0 & 2.731 & 0 & 0 & 0 & 0 & 0 & 0 & 0 & 0 \\
\hline 28 & 0 & 0 & 0 & 0 & 0 & 0 & 0 & 0 & 0 & 0 \\
\hline
\end{tabular}

...Table S2. 

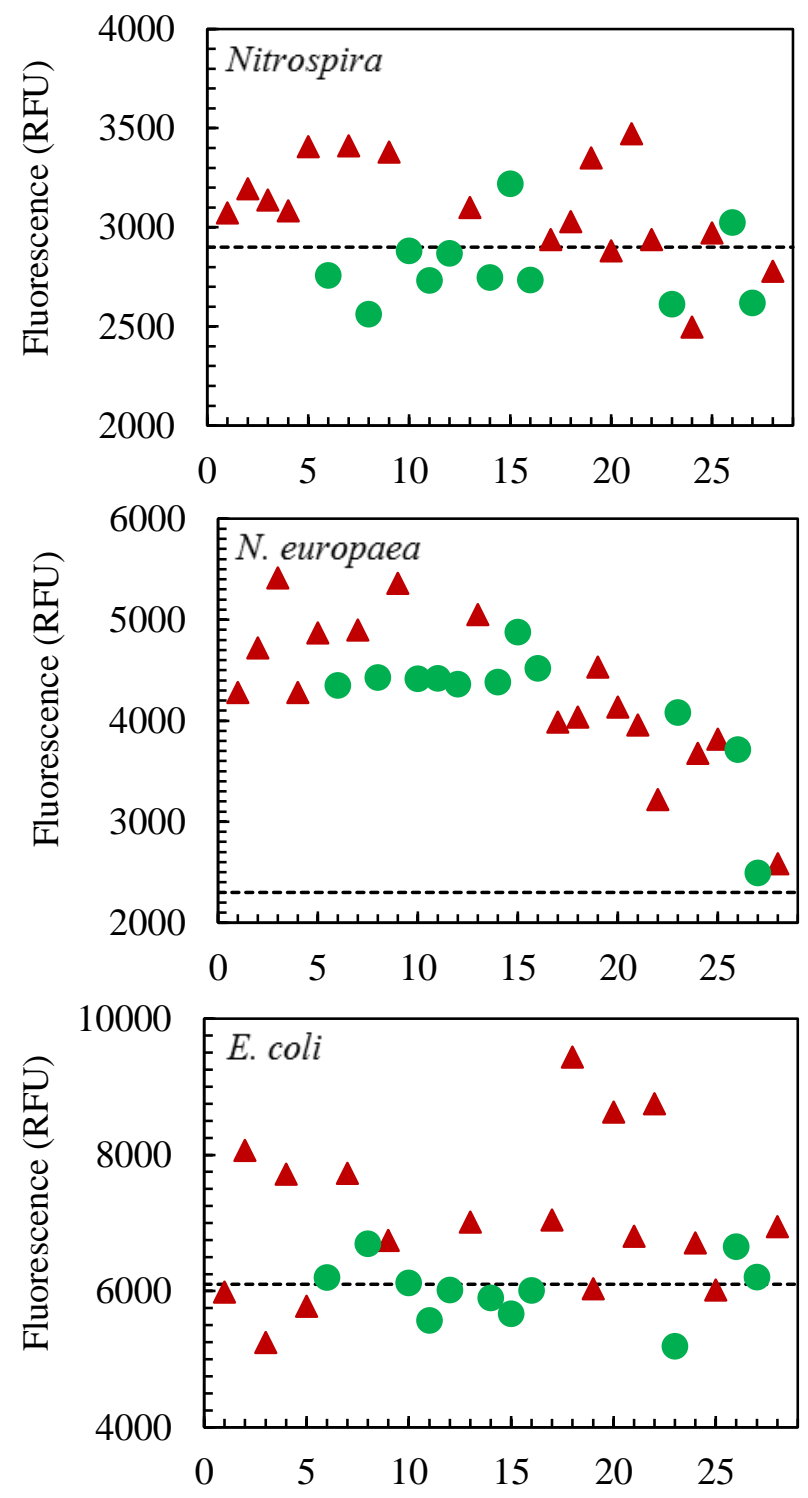

Figure S1. Fluorescence comparison for each bacterial strain (fluorescence signals are plotted using the data shown in Table S1). The dotted line represents the control signal (i.e., addition of Milli-Q water instead of WW) for each bacterial strain. The red triangles identify those WW samples that failed the SNR batch test (i.e., had \% SNR difference greater than zero). The green circles identify those WW samples that passed the SNR batch test (i.e., had \% SNR difference less than zero). Figure continued on next page. 

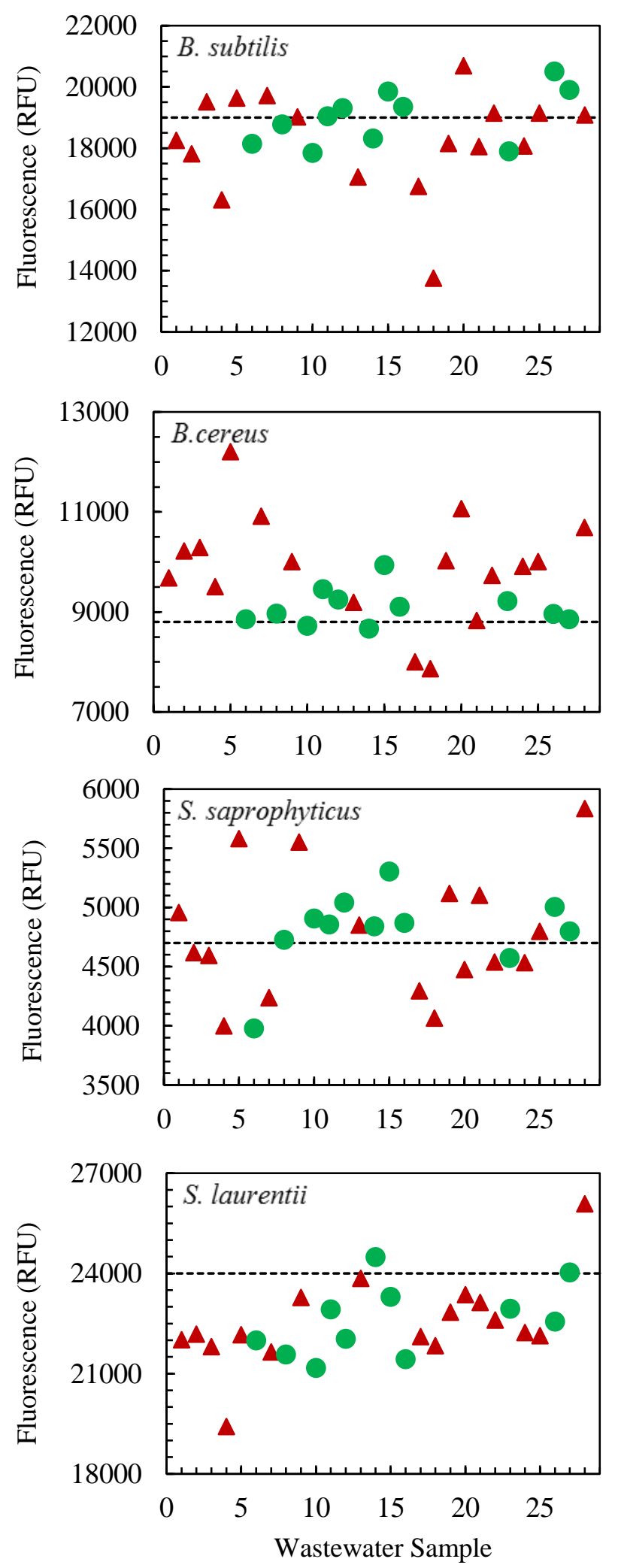

\section{...Figure S1.}




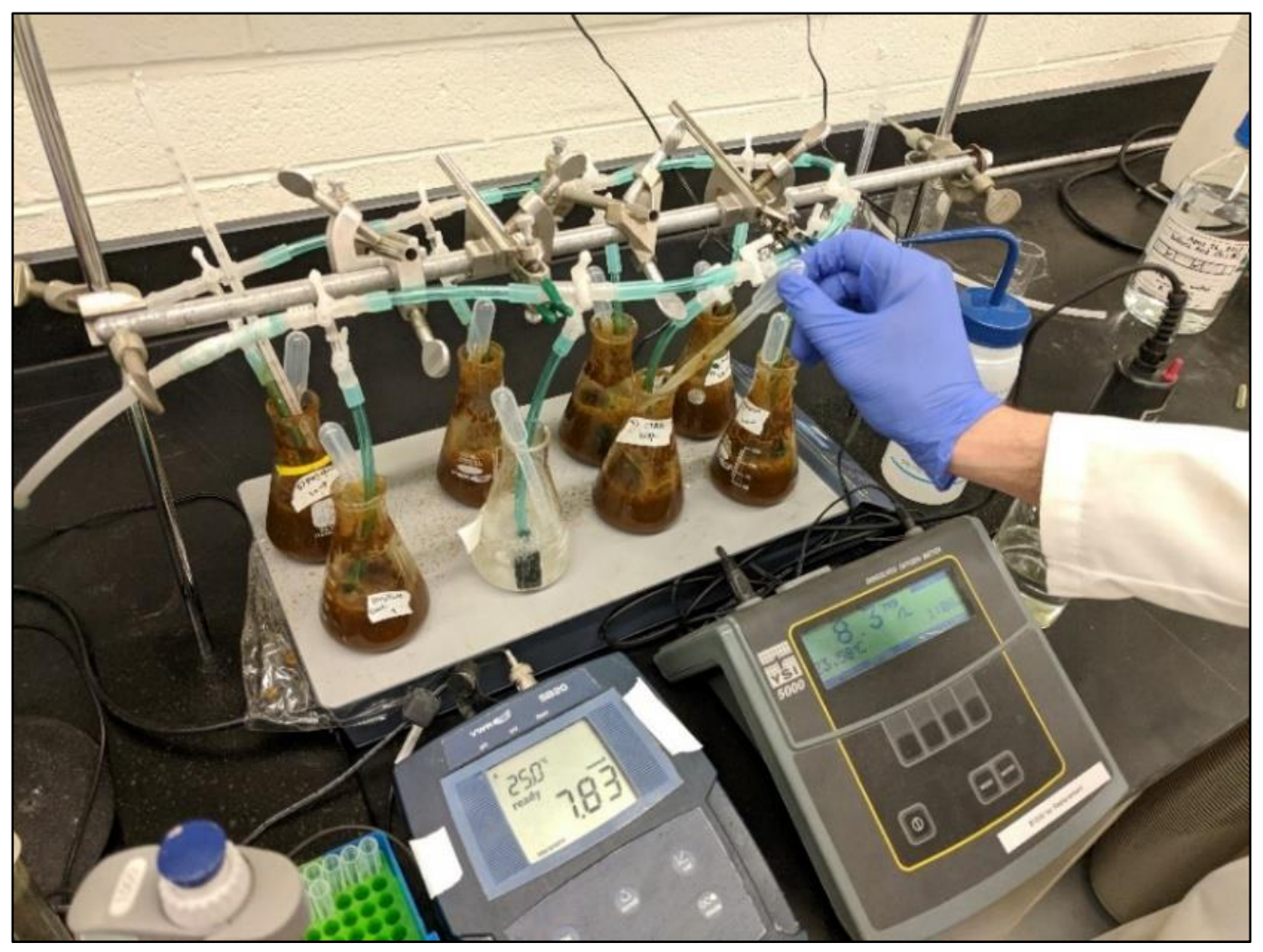

Figure S2. SNR experimental setup: $\mathrm{pH}$ meter, dissolved oxygen meter, seven $100 \mathrm{~mL}$ Erlenmeyer flasks containing return activated sludge, mineral medium, COD medium, and the corresponding WW sample. This figure shows a user sampling an SNR batch reactor for measurement of nitrite$\mathrm{N}$ and nitrate-N concentrations. The clear Erlenmeyer flask was not used in this study; typically, this flask would represent the SNR control and would look identical in color to the other flasks (Milli-Q water exposure instead of a WW sample). 

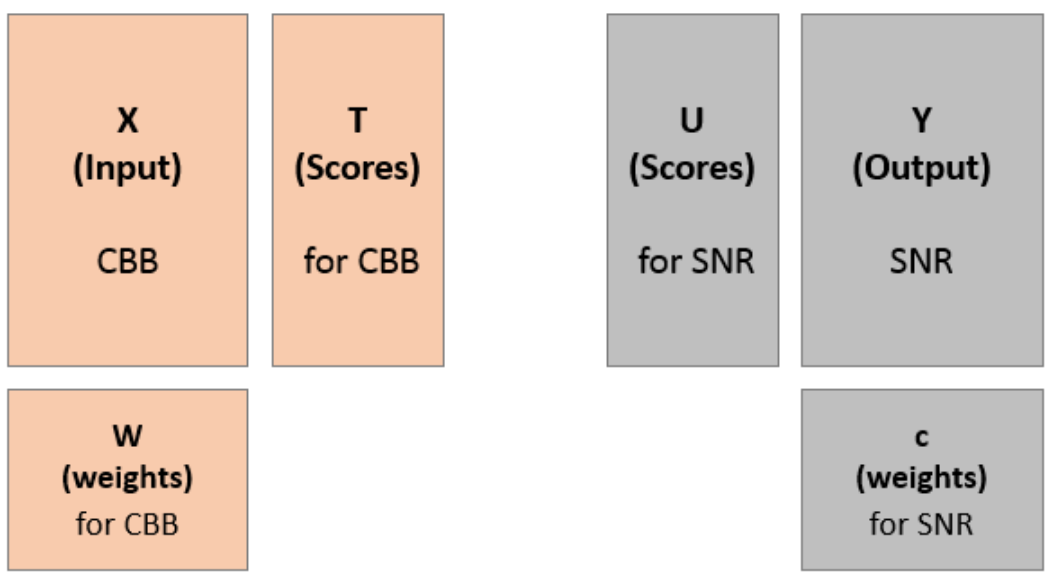

Figure S3. CBB to SNR PLS Model Setup. The X space (input) corresponds to the CBB fluorescence data. The weights (W) and scores (T) for the CBB fluorescence data are shown to the bottom and right of the $\mathrm{X}$ space (typical layout), respectively. The $\mathrm{Y}$ space corresponds to the $\%$ SNR difference data. The weights (c) and scores (U) for the \% SNR difference data are shown to the bottom and left of Y space (typical layout), respectively. PLS modeling is used to calculate the values present in the $\mathrm{W}, \mathrm{T}, \mathrm{c}$, and $\mathrm{U}$ spaces.
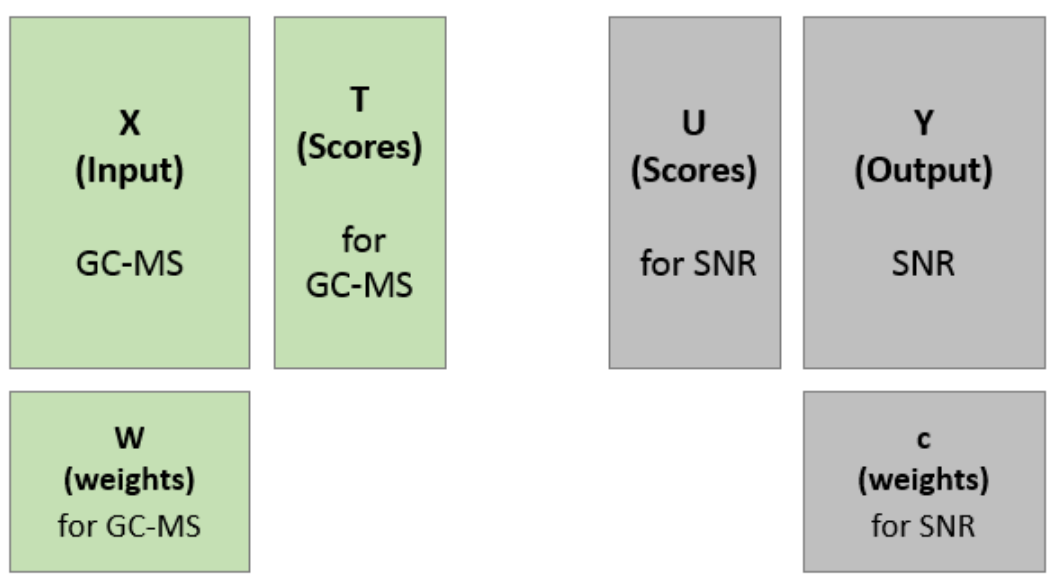

Figure S4. GC-MS to SNR PLS Model Setup. The X space (input) corresponds to the GC-MS data. The weights (W) and scores (T) for the GC-MS data are shown to the bottom and right of the $\mathrm{X}$ space (typical layout), respectively. The Y space corresponds to the \% SNR difference data. The weights (c) and scores (U) for the \% SNR difference data are shown to the bottom and left of Y space (typical layout), respectively. PLS modeling is used to calculate the values present in the W, $\mathrm{T}$, c, and $\mathrm{U}$ spaces. 

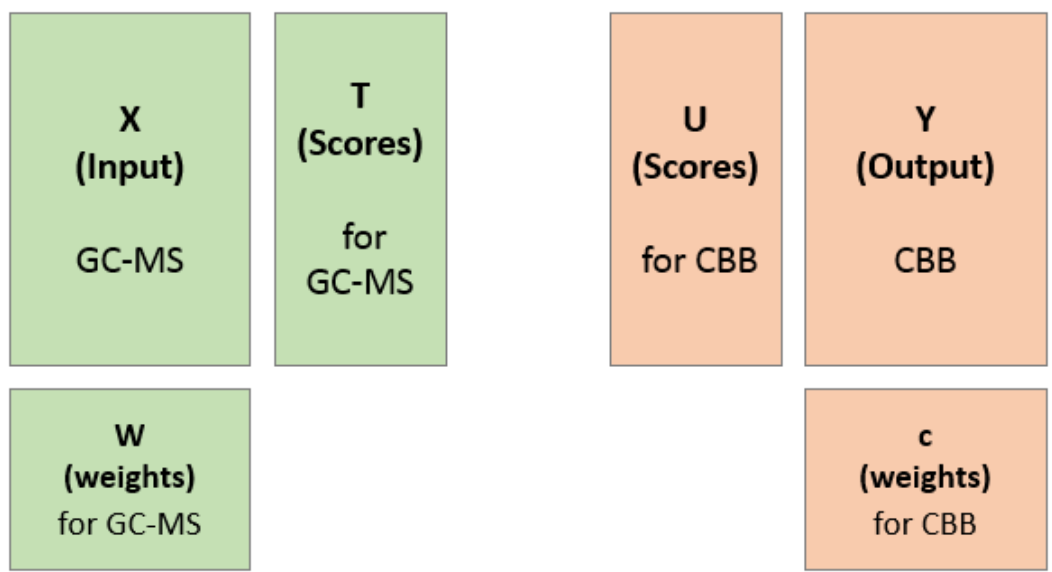

Figure S5. GC-MS to CBB PLS Model Setup. The X space (input) corresponds to the GC-MS data. The weights (W) and scores (T) for the GC-MS data are shown to the bottom and right of the $\mathrm{X}$ space (typical layout), respectively. The $\mathrm{Y}$ space corresponds to the $\mathrm{CBB}$ panel fluorescence data. The weights (c) and scores (U) for the CBB panel fluorescence data are shown to the bottom and left of Y space (typical layout), respectively. PLS modeling is used to calculate the values present in the $\mathrm{W}, \mathrm{T}, \mathrm{c}$, and $\mathrm{U}$ spaces.

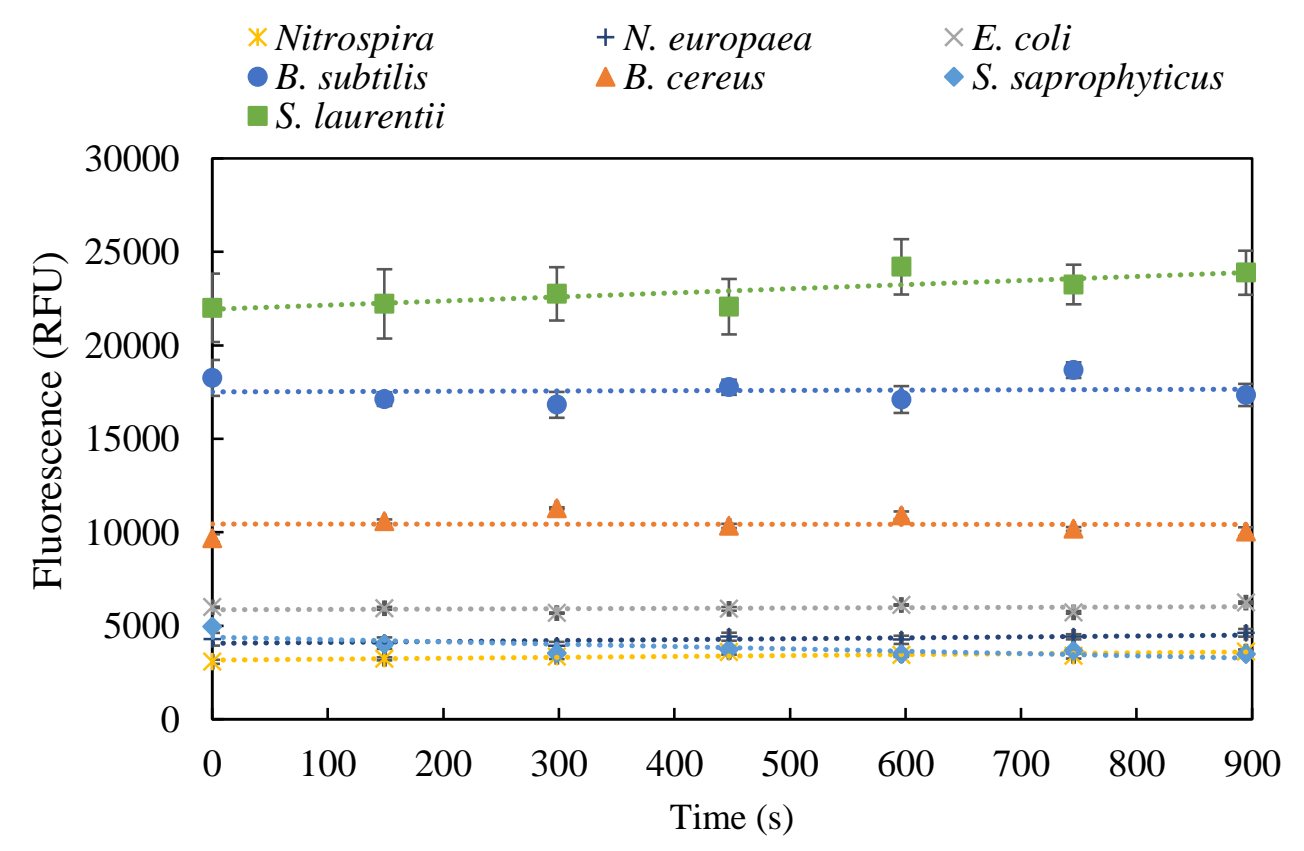

Figure S6. An example of the stability of the fluorescence signals over 15 minutes for all seven strains used in the CBB panel exposed to WW \#1. 


\begin{tabular}{|c|c|c|c|c|c|c|c|c|c|c|c|c|c|c|}
\hline \multirow{2}{*}{$\begin{array}{l}\text { Number of principal } \\
\text { components used }\end{array}$} & \multicolumn{2}{|c|}{ Nitrospira } & \multicolumn{2}{|c|}{ N. europaea } & \multicolumn{2}{|c|}{ E. coli } & \multicolumn{2}{|c|}{ B. subtilis } & \multicolumn{2}{|c|}{ B. cereus } & \multicolumn{2}{|c|}{ S. saprophyticus } & \multicolumn{2}{|c|}{ S. laurentii } \\
\hline & $\mathrm{R}^{2}$ & $Q^{2}$ & $\mathrm{R}^{2}$ & $Q^{2}$ & $\mathrm{R}^{2}$ & $Q^{2}$ & $\mathrm{R}^{2}$ & $Q^{2}$ & $\mathrm{R}^{2}$ & $\mathrm{Q}^{2}$ & $\mathrm{R}^{2}$ & $\mathrm{Q}^{2}$ & $\mathrm{R}^{2}$ & $\mathrm{Q}^{2}$ \\
\hline 1 & 0.74 & 0.16 & 0.52 & -0.02 & 0.70 & 0.09 & 0.44 & -0.17 & 0.57 & -0.15 & 0.64 & -0.52 & 0.51 & -0.73 \\
\hline 2 & 0.88 & 0.24 & 0.77 & -0.05 & 0.86 & 0.20 & 0.82 & -0.49 & 0.78 & -0.17 & 0.88 & -0.43 & 0.88 & -0.46 \\
\hline 3 & 0.97 & 0.23 & 0.95 & 0.03 & 0.94 & 0.23 & 0.91 & -0.45 & 0.91 & -0.31 & 0.97 & -0.35 & 0.95 & -0.27 \\
\hline 4 & 0.99 & 0.29 & 0.99 & 0.04 & 0.98 & 0.24 & 0.99 & -0.55 & 0.98 & -0.29 & 0.99 & -0.17 & 0.98 & 0.01 \\
\hline 5 & 1.00 & 0.36 & 1.00 & 0.04 & 1.00 & 0.27 & 0.99 & -0.62 & 0.99 & -0.32 & 1.00 & -0.10 & 1.00 & 0.11 \\
\hline
\end{tabular}

Table S3. $\mathrm{R}^{2}$ and $\mathrm{Q}^{2}$ values for different principal components for seven separate GC-MS to bacterial strain fluorescence PLS models; the same GC-MS data as shown in Figure S2 was correlated with the fluorescence signals of each bacterial strain separately using PLS modeling.

\begin{tabular}{ccc}
\hline Number of principal & \multicolumn{2}{c}{ Nitrospira \& E. coli } \\
\cline { 2 - 3 } components used & $\mathrm{R}^{2}$ & $\mathrm{Q}^{2}$ \\
\hline 1 & 0.49 & 0.05 \\
2 & 0.73 & 0.14 \\
3 & 0.81 & 0.22 \\
4 & 0.91 & 0.20 \\
5 & 0.94 & 0.24 \\
\hline
\end{tabular}

Table S4. $\mathrm{R}^{2}$ and $\mathrm{Q}^{2}$ values for different principal components for the GC-MS to CBB PLS model, where the CBB panel fluorescence data included only the fluorescence signals from Nitrospira and E. coli. The same GC-MS data as shown in Figure S2 was used. 